of the microcapsules from $\sim 4.5 \mu \mathrm{m}$ to $\sim 3 \mu \mathrm{m}$ and significantly increases the structural integrity of the capsules, which is attributed to the increase in wall thickness that accompanies the shrinking. They verified the structural integrity of the thermallytreated microcapsules by noting the percentage of capsules that were not deformed upon ingestion by cells; the capsules with thicker walls are reinforced and thus are more suited to the delivery of encapsulated materials.

Using optical transmission microscopy, the researchers demonstrated the activation of a microcapsule inside a cancer cell using a laser with a wavelength of $830 \mathrm{~nm}$ and an incident intensity of $50 \mathrm{~mW}$. The capsules had silver nanoparticles (diameter $>20 \mathrm{~nm}$ ) embedded in the walls, but had no encapsulated material. Silver nanoparticles were chosen in part because they absorb the $830 \mathrm{~nm}$ light and in part because they provide dark contrast in the optical transmission microscope. The researchers also performed release experiments on AF-488 dextran encapsulated microcapsules that had gold or gold sulfide nanoparticles embedded in the walls. These studies showed that ingested living cells adhere to the substrate both before and after the release of the encapsulated polymer, demonstrating that this method is feasible for the delivery of materials into specific cells. The studies also showed that microcapsules that were merely adhered to the outer membrane were lifted up and away from the cells during illumination and were not disrupted, demonstrating that only the ingested microcapsules release their encapsulated material upon irradiation.

THAD AWEEKA

\section{Pitting Sites on Al Controlled by Microcontact Printing Using Poly(chloroprene) Rubber Mask Film}

The arrangement of uniform tunnel pits at regular intervals is essential to maximize the surface area of $\mathrm{Al}$ electrodes for electrolytic capacitors. However, tunnel pits obtained by conventional anodic etching are not regularly distributed on an $\mathrm{Al}$ foil. Recently, K. Nishio and colleagues at Tokyo Metropolitan University developed a method to precisely control the initiation sites of tunnel pits on $\mathrm{Al}$.

As described in their article in the September issue of Electrochemical and Solid-State Letters (p. B39; DOI: 10.1149/ 1.2214364), the researchers used poly (chloroprene) rubber (CR) as a masking material for the elastomeric stamp to control the pitting sites on $\mathrm{Al}$. The researchers first cast a prepolymer of poly(dimethyl- siloxane) (PDMS) on a metal mold with an ideally ordered array of convex protrusions on its surface and cured it for $12 \mathrm{~h}$ at room temperature. After they mechanically detached the PDMS hole array (stamp) from the metal mold, the researchers dipped the stamp in a $1 \mathrm{wt} \%$ toluene solution of CR and pulled it at a constant rate. When the toluene evaporated, a thin CR film was formed on the stamp. They then transferred the CR film on the stamp to an Al foil through physical contact and detachment since the adhesion of $\mathrm{CR}$ to $\mathrm{Al}$ is much stronger than to PDMS. They then etched the Al foil covered with the patterned $\mathrm{CR}$ film in $\mathrm{HCl}$ solution under a constant dc current condition. The CR film printed on the $\mathrm{Al}$ foil masks against the anodic etching of the $\mathrm{Al}$ so that no pits are initiated on the CR film. Using this method, the researchers fabricated uniform pits arranged in an ideal array over the $\mathrm{Al}$ film with the same $\langle 100\rangle$ orientation, a width of $\sim 3 \mu \mathrm{m}$, a depth of $\sim 1.5 \mu \mathrm{m}$, and an interval of $5.0 \mu \mathrm{m}$. While the width and interval of the pits can be precisely controlled, the researchers could not control the depth of the pits, because prolonged etching induces a substantial dissolution of the sidewalls of the pits. Further study is needed to make the pits sufficiently deep.

The researchers said that using this process, they can optimize the surface area of an $\mathrm{Al}$ electrode for electrolytic capacitors by forming ordered arrays of fine and deep tunnel pits. They also said that their technique can be applied in other electrochemical and photoelectrochemical etching of semiconductors such as $\mathrm{Si}, \mathrm{InP}, \mathrm{GaAs}$, and $\mathrm{GaN}$ for simple site control of pitting.

TAO XU

\section{Sol-Gel Hybrid Film with Benzotriazole-Doped $\mathrm{SiO}_{2}$ Nanoreservoirs Provides Corrosion Protection to Al Alloy by Controlled Release of Inhibitor}

Thin sol-gel films containing inhibitors have emerged as a potential adhesive for polymer-based coating systems with selfhealing capabilities, but the integrity of the film is affected unless the inhibitor is isolated from the matrix. The concept developed to overcome this problem is based on placing the inhibitor in a reservoir or container that would slowly release it as corrosion is initiated. The addition of nanometer-sized reservoirs ensures a homogeneous distribution of corrosion protection along the film.

Using a layer-by-layer technique to fabricate reservoirs, D.G. Shchukin of the Max Planck Institute of Colloids and Interfaces in Germany; M. Zheludkevich of the
University of Aveiro, Portugal; and their colleagues created a hybrid $\mathrm{ZrO}_{2} / \mathrm{SiO}_{2}$ solgel film in which $\mathrm{SiO}_{2}$ nanoparticles coated with polyelectrolyte layers act as nanoreservoirs of the inhibitor benzotriazole, protecting an aluminum AA2024 alloy from corrosion. The layer-by-layer technique consists of depositing layers of solutions with opposite charge, leading to a multilayer assembly in which permeability can vary depending on the nature of the solutions involved. The researchers said that if polyelectrolytes are used as a shield for the inhibitor, the fact that its permeability changes with $\mathrm{pH}$ and humidity would allow controlled release of the inhibitor, depending on the very variables that determine corrosion initiation.

As explained in the July issue of Advanced Materials (p. 1672; DOI:10.1002/ adma.200502053), the research team, led by H. Möhwald of MPI, deposited positively charged polyethyleneimine (PEI) on the negatively charged $\mathrm{SiO}_{2}$ nanoparticles, with subsequent layers of polysterene sulfonate (PSS) and benzotriazole, resulting in a multilayer structure of the form PEI/PSS/inhibitor/PSS/inhibitor. In order to evaluate the corrosion-protection abilities of the nanoreservoir-doped film, the researchers compared its performance in a $\mathrm{NaCl}$ aqueous solution with an undoped $\mathrm{ZrO}_{2} / \mathrm{SiO}_{2}$ film and a film containing freely added benzotriazole $(0.13 \mathrm{wt} \%$ and $0.63 \mathrm{wt} \%$ ). Impedance measurements along a range of frequencies revealed some corrosion activity occurring in both films with free inhibitor, showing a decrease of one order of magnitude in the resistance of the film containing $0.13 \mathrm{wt} \%$ benzotriazole, and two orders of magnitude for the one containing $0.65 \mathrm{wt} \%$ benzotriazole, when compared with the undoped film. Other signs of structural degradation were found when evaluating capacitance: after $20 \mathrm{~h}$ in the corrosive environment, the undoped film capacitance increased by one order of magnitude while the $0.13 \mathrm{wt} \%$ free-inhibitor film increased by four orders of magnitude, highlighting the damaging effect of the free inhibitor in the film.

At this point, the undoped and nanoreservoir-doped films had demonstrated similar corrosion resistance in a $48 \mathrm{~h}$ period. The added benefit of the nanoreservoirs was then confirmed after the investigators introduced an identical defect on each film. Local measurements of corrosion activity using the scanning vibrating electrode technique revealed in both cases an initial severe drop in resistance, with the undoped film showing a continuous decrease in a slower ramp. For the doped film, the severe initial drop was 
followed by a continuous increase in resistance, signifying recovery of the protective properties of the film as well as slow release of inhibitor in the nanoreservoirs. The release of inhibitor was triggered by an increase of $\mathrm{pH}$ as a result of the chemical reaction between the $\mathrm{Al}_{2} \mathrm{CuMg}$ intermetallic contained in the AA2420 alloy when dissolved in the corrosive medium.

The researchers said that the results of this investigation demonstrated that the $\mathrm{ZrO}_{2} /$ nanoreservoir-SiO film provides the best corrosion protection when the $\mathrm{SiO}_{2}$ nanoreservoirs contain two sets of PSS/inhibitor layers, ensuring stability of the film with a uniform particle distribution and self-healing capabilities. The researchers said that their future plans include controlling the rate of inhibitor release and expanding the applicability of this type of coating system using other compounds more compatible with different industrial materials.

SIARI SOSA

\section{Composite Materials Fabricated through Face-Selective Adhesion of Gold Nanoparticles on L-Cystine Single Crystals}

Y. Fujiki and colleagues at Kyushu University, Japan, have produced a method to form composite materials by using transparent hexagonal prisms of L-cystine decorated anisotropically with gold nanoparticles. They reported their findings in the July 17 issue of the Angewandte Chemie International Edition (p. 4732; DOI: 10.1002/anie. 200504212).

L-cystine single crystals, recrystallized from a $0.5 \%$ hydrochloric acid solution, were immersed in a solution of gold nanoparticles $20 \mathrm{~nm}$ in diameter. The researchers used a batch method and a mounted method, consisting of mounting a crystal in a glass capillary prior to immersion, to ensure all the faces of the crystals were equally exposed to the solution of gold nanoparticles. After washing the crystals with water, the researchers observed that only the two hexagonal faces of the prisms were stained purple, due to peaking of the surface-plasmon band of the aggregated gold nanoparticles at $700 \mathrm{~nm}$. Further characterization by electron and atomic force microscopies confirmed that gold nanoparticles were deposited only on the hexagonal surfaces of the prisms, while the rectangular faces remained smooth. The researchers attributed this anisotropic decoration to the arrangement of the L-cystine molecules in the crystals. Layered structures were formed with the zwitterionic groups exposed on the surface of the hexagonal $\{001\}$ faces. The higher density of polar residues on the hexagonal faces than on the rectangular ones enabled the gold nanoparticles to attach onto these surfaces.

The researchers concluded that with different functional groups exposed on the faces of organic single crystals, other materials could be anisotropically decorated by these or other intermolecular interactions. This may allow the preparation of a wide variety of composite materials with an organic crystal core and an anisotropic aggregation of other crystals due to attractive or repulsive interactions with the uncoated or coated faces, the researchers said.

JOAN J. CARVAJAL

\section{Micromachining of $10 \mu \mathrm{M} \mathrm{Al}-0.5 \mathrm{wt} \%$ Cu Films Achieved through Porous Anodization Technique with \\ Reduced Undercutting}

Subtractive etching is a technique used in microelectronics fabrication to pattern wide metallic lines in film substrates. Wet (chemical or electrochemical) approaches to subtractive etching are economically attractive. A mask barrier is first placed in the desired configuration, and afterwards the selected etching procedure consumes the unprotected metal. However, this method has the disadvantage of undercutting or consuming some of the metal under the masking barrier. Thus, depending on the degree of isotropy of the etching process, the desired metallic feature could completely disappear.

One approach to overcoming this isotropic effect involves anodization of aluminum-patterned films to form porous alumina outside the protected regions. Still, this approach has also been limited by undercutting as a result of lateral pore growth, which consumes part of the desired metallic material. A group of researchers from the Center of MicroEngineered Materials, University of New Mexico led by D.A. Brevnov, in collaboration with G.P. Lopez and P. Atanassov, teamed with researchers from Sandia National Laboratories (Albuquerque, NM), Intel (Rio Rancho, NM), and with support from Intel (Santa Clara, CA) to determine the optimal conditions of aluminum anodization and reported their work in the August issue of Electrochemical and Solid-State Letters (p. B35; DOI: 10.1149/1.2206007).

The researchers used Si wafers, which were covered by $600 \mathrm{~nm}$ of $\mathrm{SiO}_{2}$, on which they deposited a 9.5- $\mu$ m-thick layer of $\mathrm{Al}-0.5 \mathrm{wt} \% \mathrm{Cu}$ film. The first step to create the desired pattern was to apply a $2.2-\mu \mathrm{m}$ layer of Shipley 1818 photoresist, then grow a mask of dense barrier alumina in an aqueous solution. The wafers were then anodized at $100 \mathrm{~V}$ for $2 \mathrm{~min}$, followed by the removal of the photoresist using acetone and an oxygen plasma. The configurations patterned this way resulted in 1-mm-long, 10- $\mathrm{mm}$-wide trenches set $15 \mu \mathrm{m}$ and $20 \mu \mathrm{m}$ apart (center to center), respectively, as well as 1-mm-long and 3- $\mu \mathrm{m}$-wide trenches set $6 \mu \mathrm{m}$ and $18 \mu \mathrm{m}$ apart, respectively. At this point, the researchers anodized all films in a 3\% weight/volume oxalic acid solution $(1.5 \mathrm{pH})$ at $40^{\circ} \mathrm{C}$ and $25 \mathrm{~V}$, conditions previously determined to be optimal.

Scanning electron microscopy observations of cross sections of the patterned films showed that metallic pillars formed under the masking barrier that were narrower at the top with concave slopes. The increased consumption of the metallic film immediately under the mask barrier demonstrated that lateral porous expansion was time-dependent, said the researchers. Porous alumina formed in the unprotected areas, with a volumetric expansion limited to $20 \%$, inhibiting the appearance of cracks that are produced under conditions of higher volumetric expansion. The etch factor (i.e., the ratio of the depth of the etch to the amount of the undercut) was determined to be 2 , since the undercut of material consumed under the mask barrier was half the depth of film consumed. The researchers said that this is an improvement with respect to the standard wet chemical etching technique, which gives an etch factor of about 1.2. The researchers concluded that their technique is successful for patterning $\mathrm{Al}-0.5 \mathrm{wt} \% \mathrm{Cu}$ films. The advantages of using an economical etchant method on an industry-standard metallic film can facilitate the optimization of processes for industrial electrochemical micromachining.

SIARI SOSA

The Materials Gateway - www.mrs.org 